\title{
Broadband circular interferometric millimetre-wave ISAR for threat detection
}

\author{
S. Bertl, A. Dallinger, and J. Detlefsen \\ Technische Universität München, Lehrstuhl für Hochfrequenztechnik, Fachgebiet Hochfrequente Felder und Schaltungen, \\ 80333 München, Germany
}

\begin{abstract}
To detect threats on a person's body surface the application of millimetre-waves is possible. In order to get a view of the person from all sides either the person has to be rotated standing on a turntable (Inverse Synthetic Aperture Radar, ISAR) or a sensor is moved on a circular path around the person (Synthetic Aperture Radar, SAR). The goal of the reconstruction is to obtain information about the shape of the threats and their positions in all three dimensions. At first the reconstruction is done along range and azimuth, which span the principal surface given by the sensor configuration. This paper reports on two methods to obtain the third spatial dimension. Due to the circular shape and its 3D focussing ability of the aperture this information can be obtained by the evaluation of the image focus. Secondly, if two coherent receiving channels are used, a possibility to achieve 3D spatial resolution is the processing of the interferometric phase. A comparison between these two will be presented.
\end{abstract}

\section{Introduction}

Broadband imaging in three dimensions is feasible using the scattered field distributions measured along curved lines. As a special case also circular apertures can provide 3D spatial resolution. A circular aperture can be realised by either using an array of sensors or by moving one sensor on a circular trajectory generating a synthetic aperture of circular shape.

In general holographic data can be focused for a known aperture and reconstruction area geometry as long as the sampling constraints are met. A direct inversion scheme of the linearised inverse scattering problem can be realised e.g. by means of correlation or backprojection algorithms. These algorithms are not optimum with respect to computation ef-

Correspondence to: S. Bertl

(sebastian.bertl@tum.de) ficiency (i.e. speed) but have the advantage to work with arbitrary geometries.

In order to receive focused images of the objects placed on a person's body surface, as a first approximation a cylinder can be used as reconstruction area. This geometry is shown in Fig. 1a. One the other hand the reconstruction can also be done directly along the principal axes of the sensor, which are range and azimuth, as can be seen in Fig. 1b. It turns out that this reconstruction area is favourable when considering a three-dimensional reconstruction.

In the following after the basic considerations concerning the two-dimensional reconstructions, two approaches for an independent focussing of the third spatial dimension will be presented. The first one is the interferometry, which evaluates the phase difference of two coherent receiving channels. Secondly due to the circular shape of the aperture focussing can also be done by certain amplitude criteria. These two methods can be used separately, but they can also be joined in order to combine the specific advantages of them. For example, the limited ambiguity of the interferometric phase can be compensated by the use of an additional amplitude criterion.

All the images of reconstruction results are from measured data. The measurements were done with an FMCW-Radar with a bandwidth of $10 \mathrm{GHz}$ that is described in more detail in (Detlefsen et al., 2006).

\section{SAR-processing in two dimensions}

Most of the applications based on the SAR-principle use a linear aperture or at least can neglect or compensate the curvature of it. As a consequence focussing reduces to a twodimensional problem, since a rotational symmetry with respect to the aperture axis can be applied. Each object is located at its 2-parametric position $(u, \rho)$ where $u$ repre-

Published by Copernicus Publications on behalf of the URSI Landesausschuss in der Bundesrepublik Deutschland e.V. 


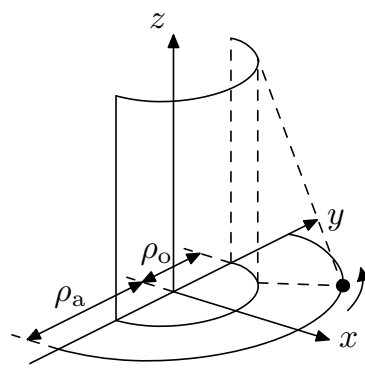

(a) Cylindrical reconstruction surface

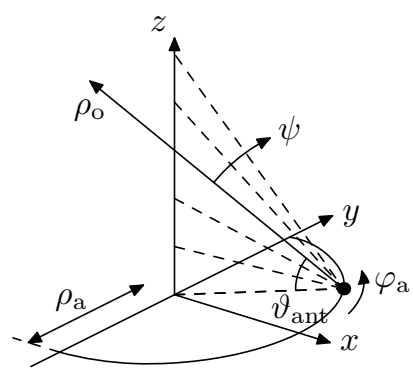

(b) Reconstruction along the principal axes
Fig. 1. SAR imaging geometry: The imaging sensor is moved on a circular path around the person. It is assumed that the person is illuminated homogeneously.

sents the azimuth variable and $\rho$ stands for range. The backprojected signal $h(u, \rho)$ then becomes

$h(u, \rho)=\int_{u_{1}}^{u_{2}} g\left(u^{\prime}, R\right) \alpha(R) \exp \left(j 2 k_{c} R\right) d u^{\prime}$

where $g$ stands for the calibrated data and $\alpha(R)$ is a weighting factor that compensates the propagation losses. $R=\sqrt{\left(u^{\prime}-u\right)^{2}+\rho^{2}}$ is the range between the antenna's aperture and the object.

For circular apertures there is no longer a rotational symmetry and therefore the SAR-reconstruction becomes a three-dimensional problem. As shown in Fig. 1 the variable for the aperture angle is denoted by $\varphi_{\mathrm{a}}$ and the radius of the aperture is $\rho_{\mathrm{a}}$. In cartesian coordinates the aperture can be written as

$\mathbf{r}_{\mathrm{a}}=(x, y, z)_{\mathrm{a}}=\left(\rho_{\mathrm{a}} \cos \left(\varphi_{\mathrm{a}}\right), \rho_{\mathrm{a}} \cos \left(\varphi_{\mathrm{a}}\right), h_{\mathrm{a}}\right)$

where the $z$-component $h_{\mathrm{a}}$ is set to zero in the following. The index $\cdot_{a}$ states that the variable describes the aperture, variables with an index ${ }_{0}$ belong to the object respectively the reconstruction area.

Since the focussing along the third spatial dimension should become independent from the first processing step, the two-dimensional SAR reconstruction is done along the mainaxes of the sensor, described by the along-track variable $\varphi_{\mathrm{o}}$ and the range variable $\rho_{\mathrm{o}}$. The angle $\psi$ stands for the offset from the main beam direction as illustrated in Fig. 1. Therefore the reconstruction area can be written as

$\mathbf{r}_{\mathrm{o}}=(x, y, z)_{\mathrm{o}}=\left(\begin{array}{l}\left(\rho_{\mathrm{a}}-\rho_{\mathrm{o}} \sin \left(\vartheta_{\mathrm{ant}}-\psi\right)\right) \cdot \cos \varphi_{\mathrm{o}} \\ \left(\rho_{\mathrm{a}}-\rho_{\mathrm{o}} \sin \left(\vartheta_{\mathrm{ant}}-\psi\right)\right) \cdot \sin \varphi_{\mathrm{o}} \\ \rho_{\mathrm{o}} \cos \left(\vartheta_{\mathrm{ant}}-\psi\right)\end{array}\right)^{\top}$

The distance $R$ between the aperture and the object position then is $R=\left|\mathbf{r}_{\mathrm{a}}-\mathbf{r}_{\mathrm{o}}\right|=f(\ldots, \psi)$. The back-projected signal can be written in the same way as (1) except that the rotational symmetry no longer holds

$h\left(\rho_{\mathrm{o}}, \varphi_{\mathrm{o}}, \psi_{\mathrm{o}}\right)=\int_{\varphi_{\mathrm{a}, 1}}^{\varphi_{\mathrm{a}, 2}} u\left(r_{\mathrm{a}}, \varphi_{\mathrm{a}}^{\prime}\right) \cdot \alpha(R) \exp \left(j 2 k_{\mathrm{c}} R\right) d \varphi_{\mathrm{a}}^{\prime}$

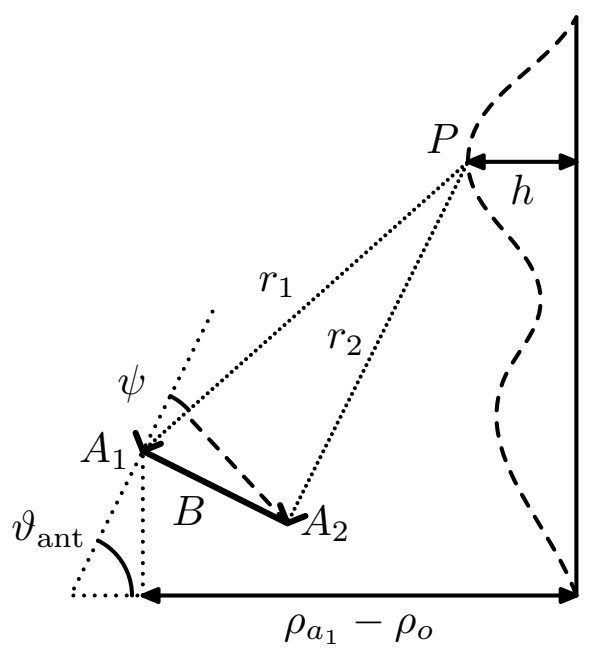

Fig. 2. Interferometric setup.

\section{Options for three-dimensional focussing}

After the SAR-reconstruction the position of the objects is determined along range $r_{\mathrm{o}}$ and cross-range $\varphi_{\mathrm{o}}$. In the following two possibilities to determine the elevation position $\psi_{0}$ will be presented.

\subsection{Interferometry}

For two coherent receiving channels separated by a distance $B$ usually called baseline the phase difference between two channels holds the information about the elevation.

Due to the physical properties of the human skin (see Dallinger et al., 2004) most of the power transmitted by a sensor below the person will be reflected and not scattered back to the receiving antennas of the sensor. As a consequence only inhomogeneities will be visible. The resulting image will consist of isolated scattering centres. Phaseunwrapping algorithms can not handle these isolated scattering centres and so the intervall of a uniquely defined phase is limited to an interval of $2 \pi$ and no absolute phase values with respect to a common reference phase can be determined.

From the two complex-valued SAR-images $h_{i}\left(r_{\mathrm{o}}, \varphi_{\mathrm{o}}\right.$, $\left.\psi_{\mathrm{o}}\right), i \in\{1,2\}$, the interferogram $v($.$) is formed by con-$ jugate complex multiplication $v()=.h_{1}(.) \cdot h_{2}(.)^{*}$. It is assumed that the position offsets of the scattering centres in the two images are smaller than one resolution element and can therefore be neglected. Figure 2 shows the geometry of the interferometric sensor with two receiving antennas $A_{1}$ and $A_{2}$ separated by the distance $B$.

In the case of one common transmitting antenna the phase difference $\phi$ obtained in the interferogram is given by

$\phi=\frac{2 \pi}{\lambda}\left(r_{2}-r_{1}\right)$ 

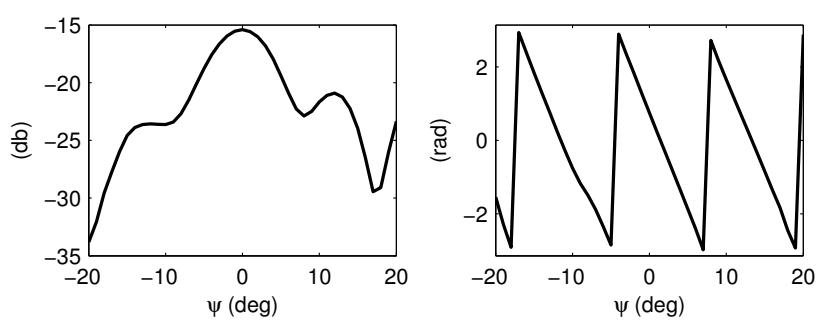

Fig. 3. Amplitude and interferometric phase along $\psi$ at the position of a metallic sphere at a position of $\psi=0^{\circ}(B=1.5 \mathrm{~cm}$, $f=[90.5 \ldots 100.5] \mathrm{GHz})$.

Since the interferometric phase is limited to $[-\pi, \pi]$ the elevation angle $\psi$ is also limited to small angles and the approximation $\sin \psi \approx \psi$ is valid. For a point scatterer located at the angle $\vartheta_{\text {ant }}-\psi_{\mathrm{o}}$ the relation between the interferometric phase and the elevation angle $\psi$ then becomes

$\Delta \phi=\frac{2 \pi}{\lambda} B \sin \Delta \psi \approx \frac{2 \pi}{\lambda} B \Delta \psi$

With a baseline $B=1.5 \mathrm{~cm}$ and a center frequency of $95 \mathrm{Ghz}$ it turns out from Eq. (6) that the unambiguousness interval $[-\pi, \pi]$ of the interferometric phase results in an unambiguousness interval of $\left[-6^{\circ}, 6^{\circ}\right]$ for the elevation angle $\psi$. Figure 3 shows that this value for $\psi$ can also be found in the reconstruction results of measured data. Here the amplitude of one channel and the interferometric phase at the $\left(\varphi_{\mathrm{o}}, \rho_{\mathrm{o}}\right)$ position of a small metallic (diameter $\approx 3 \mathrm{~mm}$ ) sphere along $\psi$ is illustrated. The characteristic property of the interferometric phase at the position of a point scatterer is, that it has a zero there.

Figure 4 shows a more complex example for the interferometric reconstruction. A person is sitting on a chair on the turntable, which results in a circular aperture, as described before. The illustration of the body surface is done by spline interploation of the weighted average of the position of scattering centres in a certain intervall $\Delta \varphi_{\mathrm{o}}$. Relevant reflection points are illustrated with dots with varying colour describing the intensity of scattering. Sub-figs. $4 \mathrm{c}$ and $4 \mathrm{~d}$ show horizontal cuts of the reconstructed model. In addition to the contour of the body a second contour appears which is an effect of the limited unambiguity of the interferometric phase. When the approximate position of the person on the turntable is known the ambiguities can be resolved by using this information.

\subsection{Options for amplitude focussing}

Besides using the interferometric phase in order to focus in the third dimension, the circular shape of the aperture also provides information about the position of the scattering centres. A possibility to determine the focus of a pixel lies in the evaluation of the gradient of its amplitude. This approach for example is used for focussing in digital still cameras as described in. The difference between a camera and a SAR

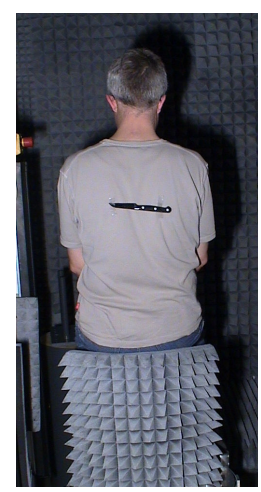

(a) photo

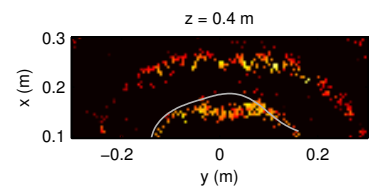

(c) horizontal cut

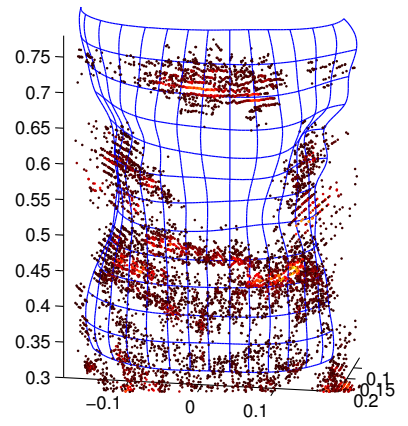

(b) three-dimensional reconstruction result
Fig. 4. Three-dimensional MMW image of a person with a horizontally orientated knife at the back.

millimetre-wave image is that for a camera only the position of the lens has to be adjusted and in a millimetre-wave image each single pixel has to be corrected seperately. An often used gradient for the purpose of focussing is the so called Sobel-gradient. In the case of focussing a pixel with respect to $\psi$ it becomes

$$
\begin{aligned}
& G_{\rho}(\psi)=\left.\left(\begin{array}{ccc}
-1 & -2 & -1 \\
0 & 0 & 0 \\
1 & 2 & 1
\end{array}\right) \otimes h(\rho, \varphi)\right|_{\psi} \\
& G_{\varphi}(\psi)=\left.\left(\begin{array}{lll}
-1 & 0 & 1 \\
-2 & 0 & 2 \\
-1 & 0 & 1
\end{array}\right) \otimes h(\rho, \varphi)\right|_{\psi} \\
& \psi_{\mathrm{opt}}=\max _{\psi} \sqrt{G_{\rho}(\psi)^{2}+G_{\varphi}(\psi)^{2}} .
\end{aligned}
$$

Another way to use the amplitude for focussing lies in the calculation of

$\psi_{\mathrm{opt}}\left(\rho_{i}, \varphi_{i}\right)=\left.\max _{\psi} h(\rho, \varphi, \psi)^{4}\right|_{r=r_{i} \pm \Delta r, \varphi=\varphi_{i} \pm \Delta \varphi}$

in an area $r=r_{i} \pm \Delta r, \varphi=\varphi_{i} \pm \Delta \varphi$ around the image position $\left(\rho_{i}, \varphi_{i}\right)$. At the $\psi$-position of a point scatterer to be focused its energy $\left(\sim h(\rho, \varphi, \psi)^{2}\right)$ is concentrated within a minimum number of pixels. Assuming that the power transmitted from the antenna to a certain area around the scattering centre remains constant and therefore also in the reconstructed image, the term $h(\rho, \varphi, \psi)^{4}$ will have its extremum at the best focused position.

Figure 5a shows the measure according to Eq. (8) along $\psi$ at the exact position of a small metallic sphere (diameter 


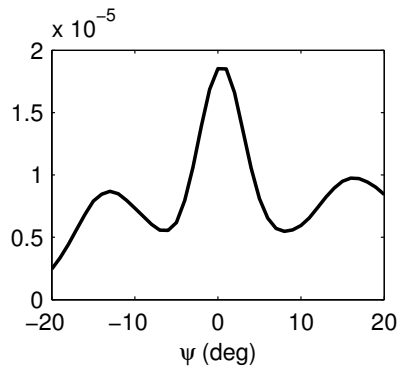

(a) measure according to eqn.

(8) at the position of a sphere

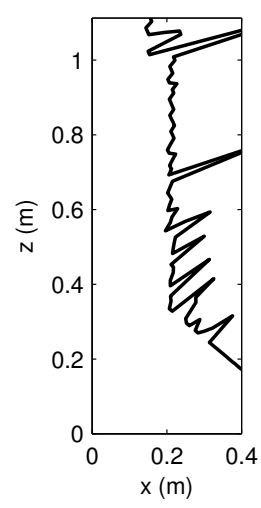

(b) $x(z=0 \mathrm{~m})$ after projection into cartesian coordinates

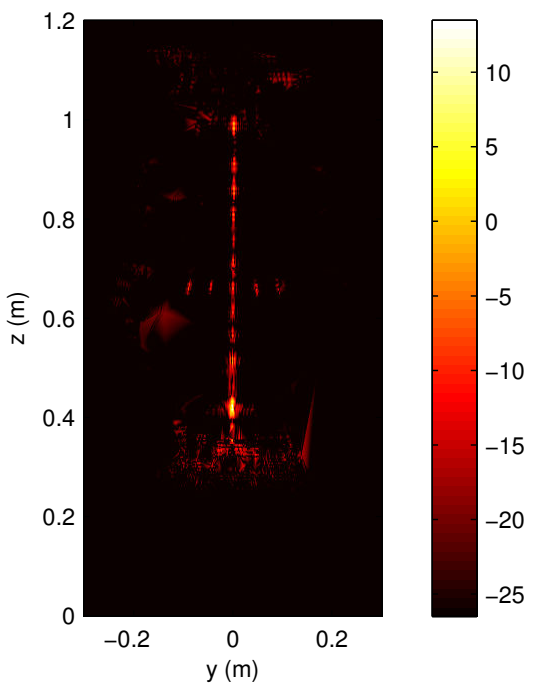

(c) photo of the (d) projection onto $y z$-plane (logarithmic measurement scaling)

scene

Fig. 5. Example for the application of an amplitude criteria for focussing along $\psi$.

$\approx 3 \mathrm{~mm}$ ) as can be seen on the photo in Fig. $5 \mathrm{c}$. The whole result of the focussing process using the amplitude criterion is shown in the other sub-figures in Fig. 5.

\section{Comparison of focussing methods}

Table 1 gives an overview of the specific properties of the two methods of focussing, namely the interferometry and the amplitude criterion, presented in the previous section.

The obvious disadvantage of the interferometry in the case of isolated scattering centres is the fact that no global phase information can be resolved. Hence only an interval of $[-\pi, \pi]$ can be used to get an unambiguous information
Table 1. Overview of the specific properties of the focussing methods presented in Sect.3.

\begin{tabular}{lll}
\hline & Interferometry & Amplitude criterion \\
\hline Uniqueness & $\begin{array}{l}\text { Uniqueness of the } \\
\text { interferometric phase }\end{array}$ & $\begin{array}{l}\text { No restriction of the } \\
\text { uniqueness }\end{array}$ \\
& $\begin{array}{l}\text { limited to }[-\pi, \pi] \\
\left.\text { (here: } \Delta \psi \approx 12^{\circ}\right)\end{array}$ & \\
\hline Processed area & $\begin{array}{l}\text { Evaluation possible } \\
\text { for each single pixel }\end{array}$ & $\begin{array}{l}\text { Averaging within an } \\
\text { area around a pixel }\end{array}$ \\
\hline Accuracy & $\begin{array}{l}\text { Accuracy can be in- } \\
\text { creased by extension } \\
\text { of the baseline (draw- } \\
\text { back: Unambiguity } \\
\text { decreases further); }\end{array}$ & $\begin{array}{l}\text { Further improvement } \\
\text { computational effort } \\
\text { Additional accuracy } \\
\text { improvement e.g. } \\
\text { by Multi-look SAR } \\
\text { processing }\end{array}$ \\
\hline $\begin{array}{l}\text { Sign of phase gives } \\
\text { direction of necessary } \\
\text { correction }\end{array}$ & $\begin{array}{l}\text { No information about } \\
\text { direction of correction }\end{array}$ \\
\hline
\end{tabular}

about the location of an object along the $\psi$-direction. In contrast the amplitude criteria are not limited in uniqueness and give a unique position over the whole range of $\psi$.

A big advantage of the interferometric focussing is the fact that the sign of the phase gives information about the direction of the necessary correction along $\psi$. Furthermore the accuracy of the interferometric phase can be increased by Multi-look processing of the SAR-data which is not explained in detail here.

Considering these facts a combination of the two focussing methods seems to make sense in the following way: The amplitude criterion is used to receive the rough position of the scattering centres along $\psi$. This compensates the unambiguousness of the interferometry. Then, in a second step the position along $\psi$ is determined more exactly using the interferometric phase.

Figure 6 finally shows the example that already appeared in Fig. 4. Here the amplitude focussing and the evaluation of the interferometric phase are joined in the way proposed before. In contrast to the processing yielding the images in Fig. 4 no previous knowledge about the position of the person is necessary here. In Fig. 6 pixels with an amplitude of $0 \ldots-35 \mathrm{~dB}$ which mostly belong to objects like the knife or the folding of the shirt are represented with colours from yellow to red. Lower amplitudes between $-35 \mathrm{~dB} \ldots-65 \mathrm{~dB}$ have grey colour. These points mostly belong to parts of the body surface. 


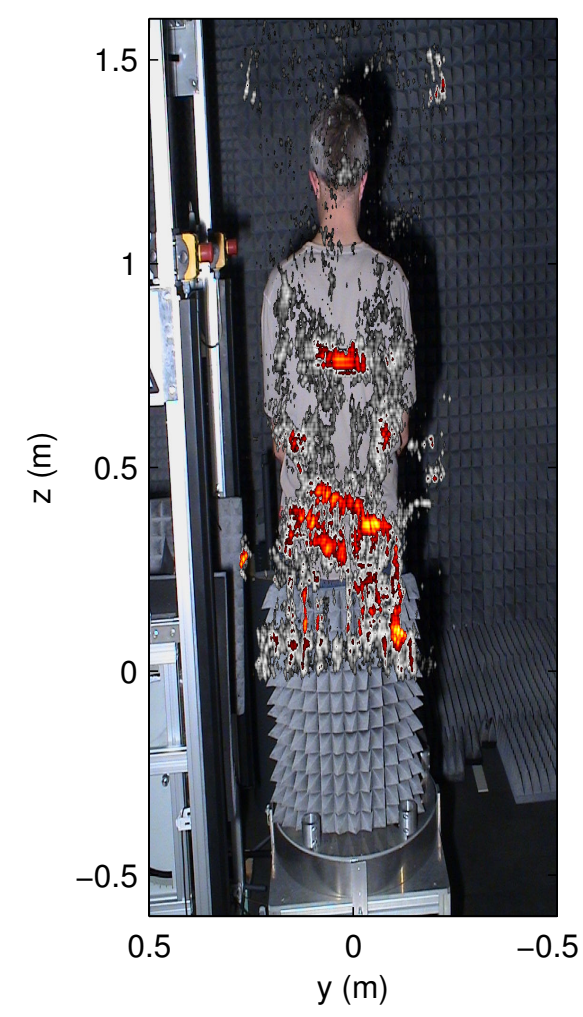

Fig. 6. Imaging results for a combined focussing using the methods two methods of Sect. 3.

\section{Conclusions}

Two methods for three-dimensional focussing of SAR images obtained by measurements along a circular aperture have been presented.

The use of the interferometric phase is similar to the way it is handled in the case of a linear aperture. Due to the application in imaging objects at the body surface of humans and the properties of human skin only isolated scattering centres can be measured. Hence no global phase information can be obtained.
To overcome this problem a second way of focussing has been presented which exploits the circular shape of the aperture by evaluating the amplitude of the scattering centres.

Finally it was shown that a combination of these two methods results in a possibility to determine a focussed image and the position of threats placed on person's body surface.

Further steps could be the discussion about the proper representation of the reconstructed image and the utilisation of faster reconstruction methods like $\omega-k$-algorithms which can also handle data of circular apertures as described in (Dallinger et al., 2005).

\section{References}

Dallinger, A., Schelkshorn, S., and Detlefsen, J.: MillimeterWave Imaging of Humans - Basic Experiments, in: IRMMW2004/THz2004, Karlsruhe, Germany, 2004.

Dallinger, A., Schelkshorn, S., and Detlefsen, J.: Efficient omegak-Algorithm for Circular SAR and Cylindrical Reconstruction Areas, Advances in Radio Science, 4, 85-91, 2005.

Detlefsen, J., Dallinger, A., Schelkshorn, S., and Bertl, S.: UWB Millimeter-Wave FMCW Radar using Hilbert Transform Methods, in: Proceedings of ISSSTA-06, 2006 IEEE Ninth International Symposium on Spread Spectrum Techniques and Applications, Manaus, Brazil, 2006.

Jie, H., Rongzhen, Z., and Zhiliang, H.: Modified fast climbing search auto-focus algorithm with adaptive step size searching technique for digital camera, Consumer Electronics, IEEE Transactions on, 49, 257-262, 0098-3063, 2003.

Rosen, P. A., Hensley, S., Joughin, I. R., Li, F. K., Madsen, S. N., Rodriguez, E., and Goldstein, R. M.: Synthetic aperture radar interferometry, Proceedings of the IEEE, 88, 333-382, 0018-9219, 2000.

Soumekh, M.: Synthetic Aperture Radar Signal Processing with Matlab Algorithms, John Wiley \& Sons, Inc., 1999.

Ulander, L. M. H., Hellsten, H., and Stenstrom, G.: Syntheticaperture radar processing using fast factorized back-projection, Aerospace and Electronic Systems, IEEE Transactions on, 39, 760-776, 0018-9251, 2003 